\title{
Preparation and Experimental Research of Nitrification Biochar
}

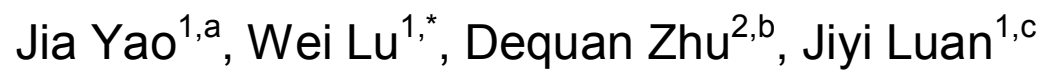 \\ 1.College of Mechanical Engineering, Jiamusi University, 154007, Jiamusi, China; \\ 2.College of Science, Jiamusi University,154007, Jiamusi, China. \\ *Corresponding author: lujunqi2001@163.com \\ aiiahuiyuyao@163.com, ${ }^{b}$ 270313501@qq.com, ${ }^{c}$ 453736626@qq.com
}

\begin{abstract}
Keywords: Biomass waste; Nitrification biochar; Biochar returning field
Abstract: Biochars, taking the biomass wastes as raw materials, can be obtained by using biomass pyrolysis technology. Biochar is favorable for environmental protection and can be taken as soil amendment, but the nutrient elements of biochar itself are poor, especially nitrogen element. Three biochars (corn stalk biochar, rice straw biochar and mushroom stuff biochar) based on different biomass wastes have been prepared at $300{ }^{\circ} \mathrm{C}$ pyrolysis temperature, then blending, adsorption and reaction methods have been taken to prepare nitrification biochars for improving the nitrogen element contents. According to the parameter variations of mass change ratios, $\mathrm{pH}$ values, SEM observations, the effectiveness for the nitrogen element adding of the three methods have been compared. Based on this research foundation, the further research of the preparation of biochar-based fertilizer and the biochar returning field technology can be pushed to promote sustainable agricultural eco-system and agricultural production.
\end{abstract}

\section{Introduction}

Mankind is facing the energy shortage situation, but biomass wastes as one kind of resources still exist insufficient use. Biomass pyrolysis technology has been used for the processing of biomass waste, the resulting liquid and gaseous substances can be used as fuel, the resulting solid material is called "biochar". Biochar has drawn considerable scientific attention due to its potential in climate change mitigation, waste management, soil fertility enhancement and crop growth promotion. The environmental benefits of biochar application to soils are associated with increased carbon (C) sequestration and suppression of greenhouse gas (GHG) emissions, while agronomic benefits include improving soil structure and porosity, increasing surface areas, water holding and cation exchange capacities (CEC)[1].

Large amount of nitrogen nutrient loss exist in farmland worldwide, espcially in China, causing resources waste and enviornment pollution. As a soil amendment, biochar has been shown to reduce nutrient leaching, immobilize heavy metals[2,3], and increase water retention and microbial activity when cultivating into soils[4]. But nutrient deficiencies of biochar are inherent, adding nitride element to biochar can give more additional nitrogen which is suitable for field crops growth, while achieving sustained-release of nitrogen element, thus can be taken as an attempt to improve the nitrogen element use efficiency. Corn stalks biochar(CSB), rice straw biochar(RSB), mushroom stuff biochar(MSB) have been prepared by pyrolysis. Three methods, including blending, adsorption and reaction method, have been adopted for preparing nitrification biochars. Through experiments and relating examinations, the comparsions of the three methods have been done and the variation principles of nitride biochar parameters have been obtained. 


\section{Experimental}

Corn stalks(CS), rice straw(RS), mushroom stuff(MS) from northern farmland of China have been selected as the raw materials for the preparation of three kinds of biochar. The limiting oxygen pyrolysis have been carried in a muffle furnace and the condition has been set as $300{ }^{\circ} \mathrm{C}$ and $1 \mathrm{~h}$. Three kind of biochar: CSB, RSB and MSB can be obtained.Chemicals used in the experiment were ammonium nitrate (solid, 35\% nitrogen content, analytical pure), aqueous ammonia(15\% concentration).

Three methods have been used for preparing nitrification biochars: 1. Blending method (BM): a mass ratio of 7:13 of ammonium nitrate with biochar has been adopted, the blending nitrification biochars can be obtained after stirring the mixture at room temperature in a blender. 2. Adsorption method(AM):Keep the mass ratio of ammonium nitrate (solid) and biochar 7:13, making ammonium nitrate(solid) full dissolved in 100 parts distilled water, then poured biochar into the solution, keep stirring, stirring time is $30 \mathrm{~min}$ and then standing for $24 \mathrm{~h}$. After drying, the objective nitrification biochars can be obtained. 3. Reaction method(RM): The mass ratio of ammonium nitrate, biochar and aqueous ammonia is 19:13:14. Adding 30\% ammonium nitrate solution and biochar to a beaker, stirring, stirring time is $15 \mathrm{~min}$; then adding $15 \%$ aqueous ammonia solution in the mixture. Continually stirring for $30 \mathrm{~min}$ and then standing for $24 \mathrm{~h}$ to collect the products. Finally the target nitrification biochars can be obtained after drying at $55{ }^{\circ} \mathrm{C}$ in an electric heated blast oven.

The mass change ratios have been determined by JY5002 electronic scales (Shanghai Shun Yuheng Scientific Instruments Co., Ltd.). PH values have been measured by a $\mathrm{pH}$ meter detection (Shanghai World Connaught physical Optics Instrument Co., Ltd.). Morphology of nitrification biochar can be obtained by using ZEISS EVO 18 scanning electron microscope instrument(Carl Zeiss company, German). Micro- Kjeldahl method was adopted to determine total nitrogen of nitrification biochars.

\section{Results discussion and analysis}

\section{Mass Changes Ratios}

After three preparing experiments, the mass of nitriding biochar has increased in varying degrees. $\mathrm{BM}$ is just a physical mixing method, so the mass changes are simply determined by the mass of raw materials. The mass changes ratios of CSB, RSB and MSB were $26.9 \%, 25.3 \%, 30.6 \%$ through $\mathrm{RM}$, the mass changes ratios were relative smaller as $13.2 \% 15.3 \%, 21.4 \%$ respectively through AM. The mass change principles can be ascribed to the different adding contents of different preparing methods. Among the three biochars, the N-carrying capacity of MSB is the best than CSB and RSB.

\section{PH Values}

As in Fig.1, pH values of the three biochars are 8.1,8.0 and 7.7 respectively. After the preparation of $\mathrm{BM}, \mathrm{pH}$ value of the three biochars are significantly decreased, showing acidity, $\mathrm{pH}$ values are 6.4, 6.8 and 6.9 respectively. PH value of mushroom nitride biochar of blending method(BMMSB) was significantly higher than rice straw nitride biochar of blending method (BMRSB) and corn straw nitride biochar of blending method(BMCSB), showing nearly neutral. After the preparation of $\mathrm{AM}, \mathrm{pH}$ values compared with biochar itself decreased, respectively reduced to 7.1, 7.2 and 7.4, it was nearly neutral to slightly alkaline, $\mathrm{pH}$ of AMMSB was the highest. After the preparation of reaction method, $\mathrm{pH}$ values of the three kinds of nitride biochars were significant alkaline, all higher than biochar itself, were 8.3, 8.6 and 8.7 respectively, where the highest $\mathrm{pH}$ value was RMMSB. PH value of $\mathrm{RM}$ was alkaline, but $\mathrm{pH}$ value of the two other methods presented acidic, $\mathrm{pH}$ values of $\mathrm{BM}$ were the lowest. 


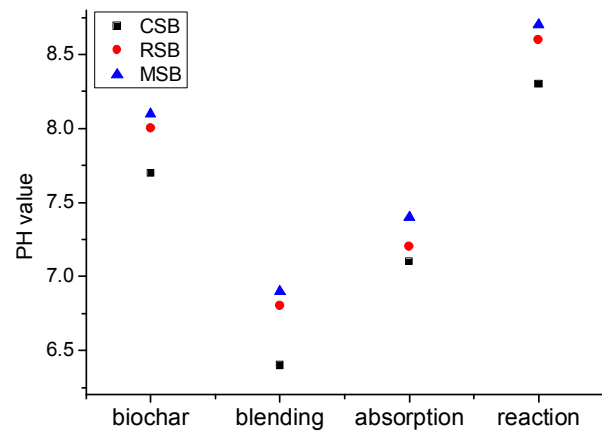

Fig.1 PH values of biochars and nitride biochars

For BM and AM, ammonium nitrate is the only reagents, ammonium nitrate is acidity, neutralizing the alkalinity of biochar, so the $\mathrm{pH}$ values decreased accordingly. Besides ammonium nitrate, aqueous ammonia was added for the reaction method, aqueous ammonia itself rendered alkaline, so $\mathrm{pH}$ values of the corresponding nitride biochar increased slightly. Obviously, all the three methods can largely change the $\mathrm{pH}$ values of nitriding biochars. In contrast, the nitrogen biochar of BM is suitable for acid crops, the nitrogen biochars of AM and RM are more suitable for alkali crops.

\section{SEM Analysis}

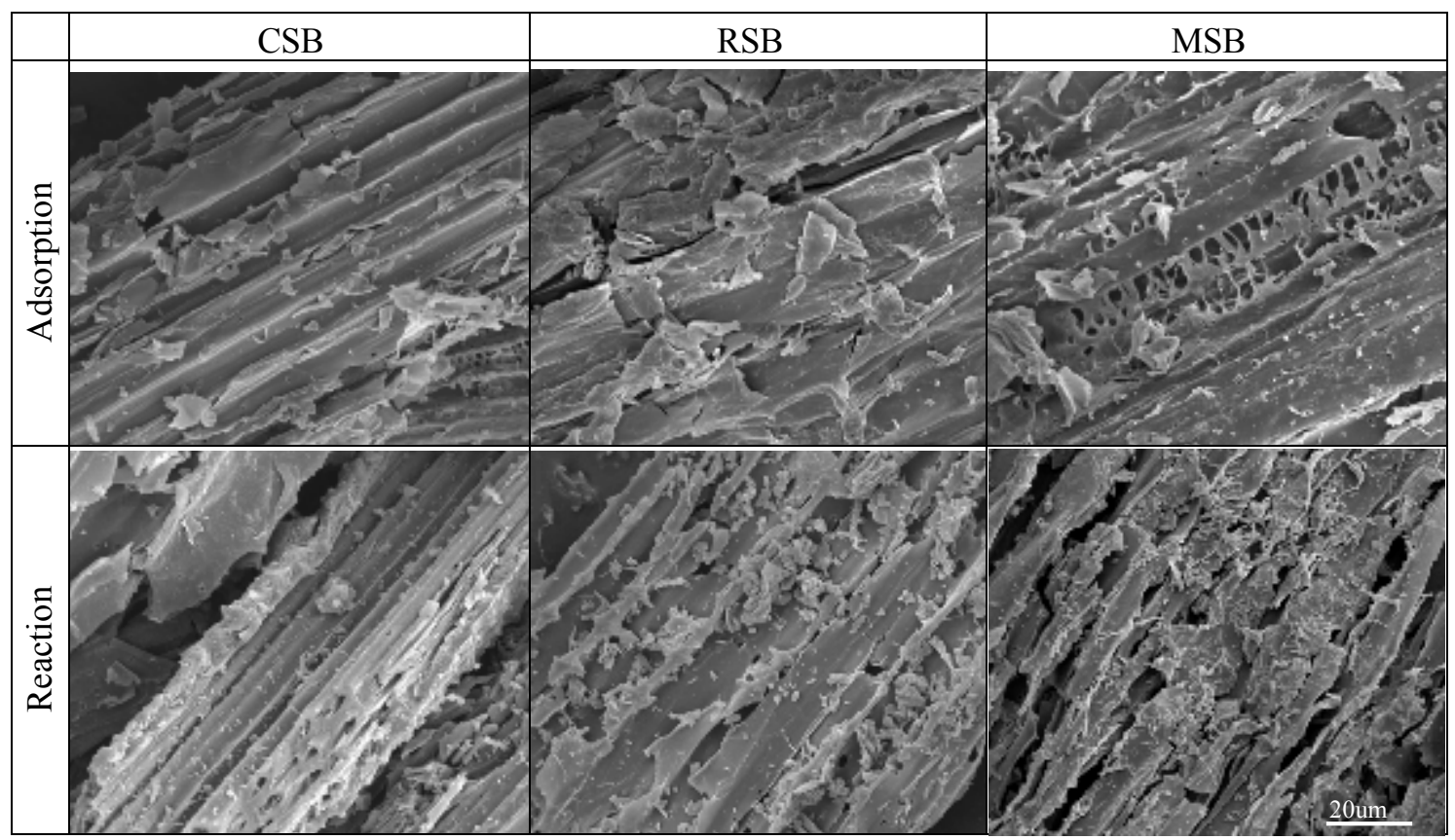

Fig.2 SEM observation of three nitrogen biochars got by AM and RM

Biochar itself has rich pores with different sizes and the pores appear a close tubular longitudinal arrangement structure. These pore structures and surfaces provide enough space for particle adsorption. The SEM observations have been obtained for the biochars got by AM and RM as in Fig.2, obvious differences exist between AM and RM. After AM, a lot of ammonium nitrate appeared in the holes and the crystallization particulate matter can be observed on the surfaces. After RM, chemical reaction takes place in biochar, ribbon-like substances exist on the side section of biochar surface. The appearance change of biochar is ascribed to the chemical reaction among biochar, ammonium nitrate and aqueous ammonia, the growth of nitrogen material can be observed in the carbon surface obviously. 


\section{Nitrogen content of different nitrified biochar}

The nitrogen contents of the nine nitrogen biochars were in Table.1. According to the data, nitrogen contents of BM is just a physical mixing method, so the nitrogen contents are simply determined by the mass of raw materials, the values of the three biochar are almost the same as $12.23 \%, 12.22 \%$ and $12.25 \%$ respectively caused by operation errors. AM first need to make ammonium nitrate(solid) full dissolved in water, then the ammonium nitrate can be absorbed effectively in the biochar holes, but the water may take nitrogen partly away, so the nitrogen content values are slightly reduced comparing with blending method, as $11.29 \%, 11.43 \%$ and $11.85 \%$ respectively. The RM values are larger than the other two methods, as $22.34 \%, 23.46 \%$ and $25.03 \%$ respectively, which is ascribed to the more $\mathrm{N}$ element adding obtaining by the raw materials and $\mathrm{N}$-carrying capacity is more effective by the chemical reaction.

Table.1 Nitrogen contents of the nine samples

\begin{tabular}{cccc}
\hline & $\mathrm{CSB}(\%)$ & $\mathrm{RSB}(\%)$ & $\mathrm{MSB}(\%)$ \\
\hline $\mathrm{BM}$ & 12.23 & 12.22 & 12.25 \\
\hline $\mathrm{AM}$ & 11.29 & 11.43 & 11.85 \\
\hline $\mathrm{RM}$ & 22.34 & 23.46 & 25.03 \\
\hline
\end{tabular}

\section{Conclusions}

Adopting BM, AM and RM to prepare nitrification biochars, the changes parameters of various raw material biochar after nitrification have been researched. The mass change ratios of CSNB, RSNB and MSNB under RM and AM show the same trend, MSB have the best N-carrying ability. PH values decreased for $\mathrm{BM}$ and $\mathrm{AM}$, but increased slightly for $\mathrm{RM}$, due to the difference of preparation method. The results of SEM further confirmed the existence of nitrogen in biochar after $\mathrm{AM}$ and RM processes, the difference is ascribed to chemical reaction or physical adsorption. The nitrogen contents of BM is larger than AM, but RM has the largest values, which is ascribed to the more $\mathrm{N}$ element adding of the raw materials and more effectiveness of N-carrying capacity of chemical reaction. Further research can be improved by adding more nutrient elements to biochar, and effectively push the biochar returning field. Also the effects of biochars on the metabolism of other nutrient elements and the effects on the growth of crops can be investigated in the future.

\section{Acknowledgments}

This project was funded by National Natural Science Foundation of China (51203061); the Youth Backbone Support Plan for Heilongjiang Province Universities (1254G059); the Innovation team of Jiamusi Unversity(Cxtd-2016-02), the Applications Project of Jiamusi Unversity(12Z2201523) and Jiamusi University Education Research Project Funding.

\section{References}

[1] Verheijen, F.G.A., Graber, E.R., Ameloot, N., Bastos, A.C., Sohi,S. , Knicker, H: Eur J Soil Sci 65(2014),p.22-27 .

[2] Ahmad, M., Rajapaksha, A.U., Lim, J.E: ChemospHere. 99(2014),p.19-33.

[3]Beesley, L., Moreno-Jimenez, E., Gomez-Eyles, J.L., Harris, E., Robinson, B., Sizmur, T: Environ Pollut. 159(2011),p.3269-3282.

[4] Atkinson, C.J., Fitzgerald, J.D., Hipps, N.A: Plant Soil 337(2010), p.1-18 . 